\title{
Use of String of Pearls Locking Plate System, Intramedullary Pin and Lag Screws for the Treatment of an Intra-Articular Olecranon Fracture in a Lamb
}

\author{
Darby W. Walmsley ${ }^{1}$ Tania Shaw $^{1}$ Simon T. Kudnig ${ }^{1}$ \\ ${ }^{1}$ Surgery Department, Animal Referral Hospital, Essendon Fields, \\ Melbourne, Australia \\ Address for correspondence Darby W. Walmsley, BVSc MRCVS, \\ Animal Referral Hospital, Essendon Fields, 72 Hargrave Avenue, \\ Essendon Fields, Melbourne, 3041, Australia \\ VCOT Open 2019;2:e50-e55 \\ (e-mail: darby.walmsley@gmail.com).
}

\begin{abstract}
Keywords

- olecranon fracture

- sheep

- String of Pearls

- fracture

- tension band wire
\end{abstract}

Objectives The aim of this study was to describe a case of an intra-articular olecranon fracture in a lamb repaired using a String of Pearls locking plate system, intramedullary pin and lag screws.

Materials and Methods A 6-month-old, $20 \mathrm{~kg}$, purebred male Merino lamb was referred for evaluation of a non-weight bearing lameness of the left thoracic limb of unknown duration. Orthogonal radiographic assessment of the left elbow revealed a complete, closed, long sagittal oblique, minimally displaced fracture of the olecranon involving the ulnar shaft at the level of the radial physis, extending proximally through the distal semilunar notch to the mid-body of the olecranon. The fracture was repaired using a String of Pearls locking plate system spanning both the proximal radius and olecranon, an intramedullary pin in the ulna and lag screws across the fracture line.

Results Orthogonal radiographic assessment of the left elbow at 8 weeks postoperatively revealed implant quiescence with radiographic union of the fracture site. Longterm telephone follow-up at 3 and 6 months postoperatively found the patient was no longer restricted in activity, with free access to the field, and was not displaying an overt lameness.

Clinical Significance The authors describe a novel method to treat an intra-articular in a lamb olecranon fracture in a lamb using a String of Pearls locking plate system, intramedullary pin and lag screws.

\section{Introduction}

Correction of proximal ulna and olecranon fractures is extensively described in veterinary literature. ${ }^{1-9}$ Their prevalence is, however, somewhat uncommon when compared with other antebrachial fractures. Such fractures may be non-articular or articular in nature, with the latter most common, and have been reported in dogs, ${ }^{1-3}$ cats, ${ }^{1}$ horses ${ }^{4-8}$ and a bull. ${ }^{9}$ To counteract the tensile force of the triceps brachii muscle group on the olecranon, various repair techniques, including tension band wiring (TBW) and plate osteosynthesis (PO), have been described to convert tensile forces to compressive forces while achieving interfragmentary compression and anatomical reduction. ${ }^{1,3,4}$ Open reduction and internal fixation with application of a TBW have been proposed as the treatment of choice for proximal ulna fractures in dogs, but is associated with a higher complication rate than $\mathrm{PO}^{3}$

Type of repair and implant selection is not only based on fracture configuration but is also defined by specific species characteristics, including anatomical differences and the physiological requirement for pronation and supination. The ulna bone characteristically tapers along its diaphyseal received

July 23, 2019

accepted after revision

September 24, 2019
DOI https://doi.org/

10.1055/s-0039-1700844. ISSN 2625-2325. (c) 2019 Georg Thieme Verlag KG
Stuttgart · New York

License terms

(c) (1) 
length distally, forming the metaphysis and articulation with the carpus. ${ }^{10}$ In domestic animals, the width of the ulna provides sufficient bone stock and medullary cavity for plating laterally, medially and caudally, or for application of a TBW or intramedullary pin. Small ruminants dissimilarly do not require the ability for pronation and supination, which consequently results in the development of a narrower ulna with reduced medullary canal diameter distally ${ }^{10}$ (-Fig. 1). Distinct to the horse, the proximal ulna diaphysis is well developed but is fused to the radius, with the distal ulna absent and no carpal articulation. ${ }^{10}$ The anatomical variations between domestic animals and that of the equine and ovine antebrachium are important for surgical planning and appropriate implant selection.

Antebrachial fractures in small ruminants are infrequently reported despite being well-documented in cattle. ${ }^{9,11}$ The decision to treat a fracture in small ruminants is often made by considering economic or genetic value of the animal, cost of treatment, morbidity and mortality rates and fracture location and configuration. ${ }^{11}$ Literature describes the use of casting or splinting, ${ }^{11}$ internal plate fixation, ${ }^{11}$ semicircular external fixation ${ }^{12}$ and combined positive profile transfixation pins
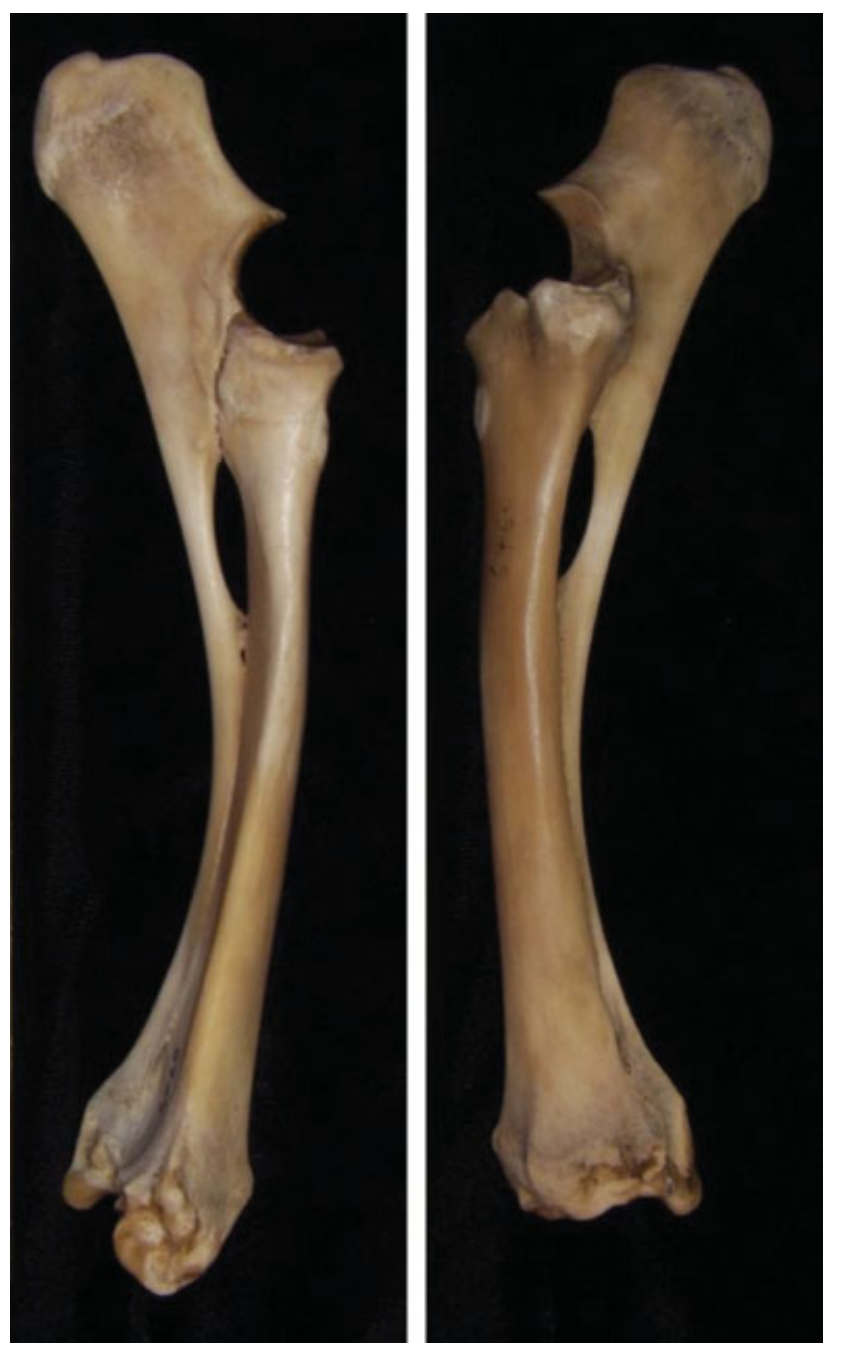

\section{Diagnostic Imaging and Surgical Planning}

Orthogonal radiographic (craniocaudal and mediolateral projections) assessment of the left elbow revealed a complete, closed, long sagittal oblique, minimally displaced fracture of the olecranon involving the ulnar shaft at the level of the radial physis, extending proximally through the distal semilunar notch to the mid-body of the olecranon (-Fig. 2A, 2B). Additionally, a small lateral humeral epicondylar avulsion fracture was also apparent (-Fig. 2B). Secondary proliferative callus formation was apparent over the caudoproximal aspect of the olecranon (-Fig. 2A), suggestive of fracture chronicity. The proximal ulna epiphyseal plate remains open.

The medullary canal of the ulna is incomplete and remained radiographically open for $8.8 \mathrm{~cm}$ from the most proximal aspect of the olecranon to the proximal ulna diaphysis, which is the most distal aspect of the medullary canal, before fusing with the caudal aspect of the proximal radius. The greatest width of the medullary canal of the ulna was $3.8 \mathrm{~mm}$.

\section{Surgical Technique}

The lamb was pre-medicated with methadone $(0.2 \mathrm{mg} / \mathrm{kg}$ intramuscularly; Methadone, Ilium, Troy Laboratories Australia Pty Ltd, Australia). Anaesthesia was induced with propofol (4 mg/kg intravenously [IV]; Lipuro $1 \%$, Braun Australia Pty Ltd, Bella Vista NSW, Australia) and maintained with isoflurane (Isoflo, Zoetis Australia Pty Ltd, Rhodes NSW, Australia) in oxygen. The left thoracic limb was clipped and prepared with chlorhexidine and an alcohol solution, and the patient positioned in dorsal recumbency.

A lateral approach to the left elbow was performed. The large amount of proliferative callus was debrided, allowing the sheep. 


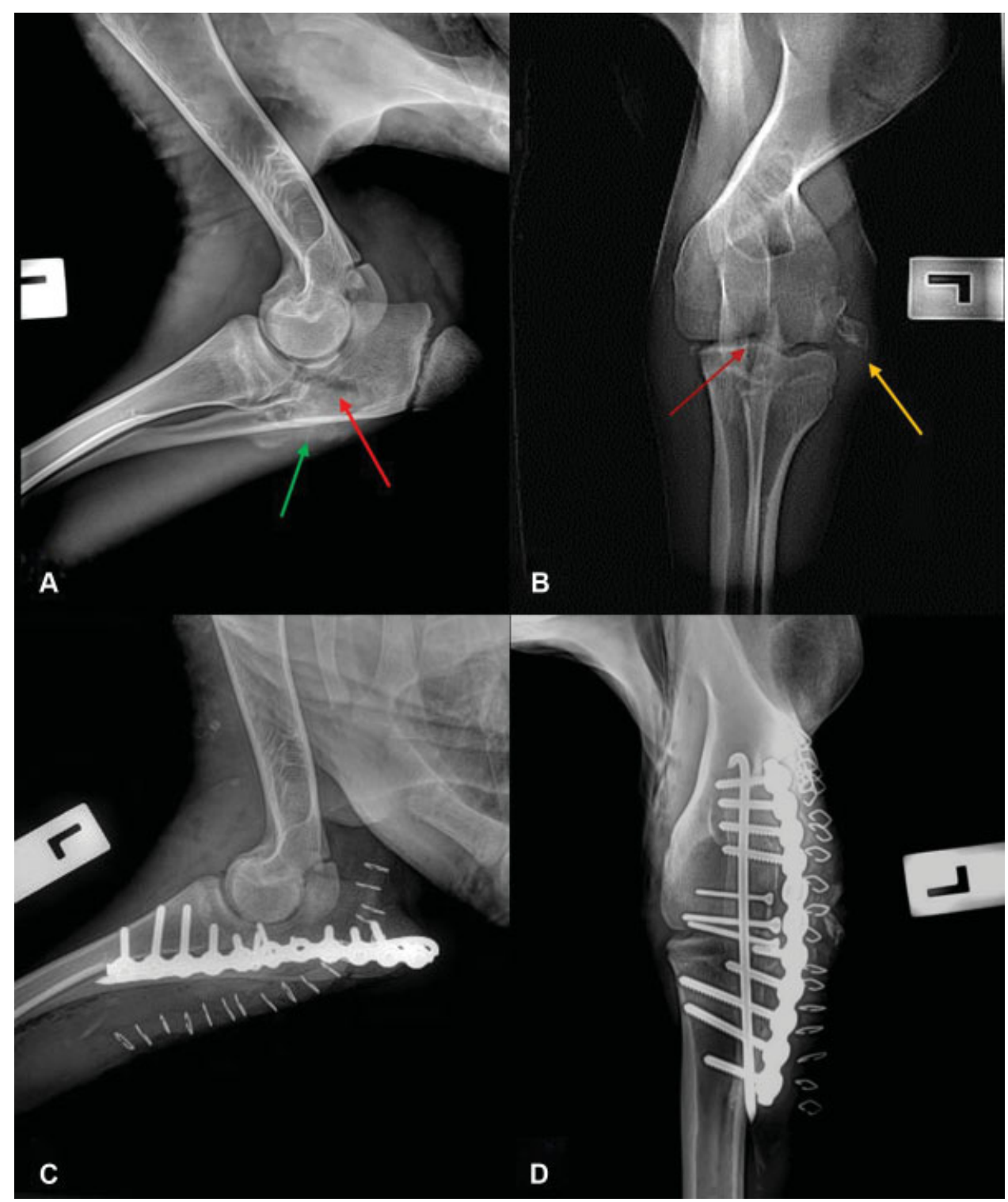

Fig. 2 Preoperative and immediate postoperative radiography (mediolateral projection, A and C; craniocaudal projection, B and D) of the left elbow. Red arrow: fracture of the olecranon involving the ulnar shaft at the level of the radial physis, extending proximally through the distal semilunar notch to the mid-body of the olecranon. Yellow arrow: small lateral humeral epicondylar avulsion fracture. Green arrow: secondary proliferative callus formation.

exposure of the fracture site. A $3 / 32$ inch $(2.4 \mathrm{~mm})$ intramedullary pin was placed normograde from the most proximal aspect of the olecranon across the fracture and into the distal ulna. The pin was observed to exit the cortex distally at the most distal aspect of the medullary canal. The fracture was reduced and maintained in reduction using pointed reduction forceps. Two $2.0 \mathrm{~mm}$ lag screws were placed in a lateral-medial direction across the fracture site. An additional $1.5 \mathrm{~mm}$ lag screw was also placed in the same direction. Due to the lack of bone stock available in the distal ulna, a $2.7 \mathrm{~mm}$ String of Pearls plate (Orthomed Australasia Pty Ltd, Mandurah, WA, Australia) was contoured over the lateral aspect of the proximal radius and lateral aspect of the olecranon and attached to the proximal radius and olecranon using $2.7 \mathrm{~mm}$ cortical screws. Two screw holes were left empty over the fracture site. The elbow was stable on palpation and when manipulated through range of motion. Based on this stability combined with the chronicity of the injury and amount of firm callus identified laterally, we elected not to explore the avulsion fracture from the lateral humeral epicondyle. A routine multilayer closure of the incision was performed.

Postoperative radiographs revealed appropriate implant positioning and anatomic fracture reduction ( - Fig. 2C, 2D).

\section{Perioperative and Postoperative Management}

Perioperative antibiotic therapy consisted of cefazolin (22 mg/kg IV; Cefazolin-AFT, AFT Pharmaceuticals, North Ryde, NSW, Australia) at least 30 minutes prior to the first incision, and every 90 minutes during surgery thereafter. Perioperative analgesia included methadone $(0.1 \mathrm{mg} / \mathrm{mg} I V)$ administered once intraoperatively. Postoperative analgesia 
consisted of methadone $(0.2 \mathrm{mg} / \mathrm{kg}$ IV q6h), meloxicam (0.1 mg/kg subcutaneously; Meloxicam, Ilium, Troy Laboratories Australia Pty Ltd, Australia) and a transdermal fentanyl patch (50 mcq/h; Durogesic 50, Janssen-Cilag Pty Ltd, Macquarie Park NSW, Australia). Pain scores were performed every 4 hours with appropriate administration of methadone as required until activation of the transdermal fentanyl patch 12 hours following application.

The patient was discharged from hospital the following day, with meloxicam $(0.1 \mathrm{mg} / \mathrm{kg}, 1.5 \mathrm{mg} / \mathrm{mL}$; Meloxicam, Ilium, Troy Laboratories Australia Pty Ltd, Australia) dispensed for oral administration during the postoperative period. The patient was to be restricted to a small pen for 8 weeks.

\section{Clinical Outcome}

The patient presented 8 weeks postoperatively for the reevaluation by their primary care veterinarian. Physical examination revealed a grade $1 / 5$ left thoracic limb lameness. Range of motion of the elbow was now considered within normal limits. Digital pressure application over the lateral humeral epicondyle, site of previous avulsion fracture, elicited a mild pain response. Orthogonal radiographic assessment of the left elbow revealed implant quiescence with radiographic union of the fracture site ( - Fig. 3 ). Indirect bone healing is evident at the site of previous lateral humeral epicondylar avulsion fracture. Ongoing thoracic limb lameness is hypothesized to be a result of periarticular inflammation associated with secondary bone healing. The patient was no longer receiving non-steroidal anti-inflammatory medication at the time of recheck.

Telephone updates were provided at both 3 and 6 months postoperatively by the client. According to the client, the patient was no longer restricted in activity, with free access to the field, and was not displaying an overt lameness. Follow-up of the patient was lost thereafter.

\section{Discussion}

Despite their uncommon prevalence, olecranon fractures are generally associated with satisfactory outcomes despite relatively high complications rates of up to $52 \%$ in dogs. ${ }^{3}$ Plate osteosynthesis is associated with a more biomechanically

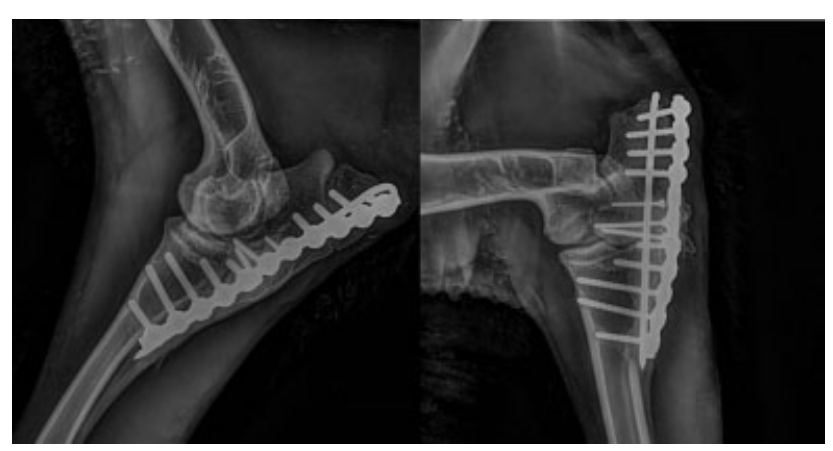

Fig. 3 Radiographs of the left elbow obtained 8 weeks postoperatively. robust construct, fewer complications and improved outcomes when compared with $\mathrm{TBW}^{3,4,7,8,14,15}$ however, the use of TBW remains advocated in some cases of non-displaced, non-articular olecranon fractures. ${ }^{11}$ Fournet and colleagues $^{3}$ hypothesized that anatomical differences of the proximal ulna between the dog and cat, as well as patient weight, are a major factor associated with selection of repair technique, emphasizing the importance of appropriate implant selection and placement.

Several classification systems for olecranon fractures have been described in humans. ${ }^{16-18}$ Currently, factors such as fracture type, location and its open verses closed status are the main characteristics that guide management of such cases in dogs. ${ }^{3}$ A universally accepted classification system has been adopted for equine ulnar fractures, ${ }^{19}$ classifying fractures as Type 1 through to Type 5 dependent on their configuration, although variations of these fracture configurations have been reported. Given the anatomical similarities between the antebrachium of the horse and sheep and the high-energy traumatic nature of ulna fractures in both species, the authors postulate that the aforementioned classification system for ulnar fractures may also be ascribed to that of small ruminants. This case report describes a fracture most closely mirroring a Type 5 olecranon fracture in a horse, as the fracture configuration includes the involvement of the ulnar shaft at the level of the radial physis that extends proximally into the semilunar notch. The results of plate fixation of Type 5 olecranon fractures in horses have most recently been described by Swor and colleagues, ${ }^{15}$ with longterm follow-up (16-146 months following surgery) revealing $85 \%$ of horses were sound and used for their intended athletic purpose. The same study ${ }^{15}$ suggested that tension band plating provides several advantages over conservative management and other internal fixation methods, including increased inherent repair stability, an anticipated decrease in degenerative changes of the joint and faster healing times. Type 5 fractures managed with TBW in five horses reported sound outcomes in three cases and one case with a fair longterm outcome. ${ }^{20}$ It is now widely recognized that TBW is associated more readily with complications than with plate fixation, particularly in adult horses due to increased risk of cyclical implant fatigue and failure of the biomechanically inferior TBW. $3,4,7,8,14,15$

While conservative therapy may be an option in patients with olecranon fractures, $\sim 90 \%$ and the vast majority of olecranon fractures in $\operatorname{dog} s^{3}$ and horses, ${ }^{19}$ respectively, have intra-articular involvement, and are therefore not amenable to the application of a cast or external fixation and require interfragmentary compression techniques. In a field setting, non-surgical management of ulna fractures may include the application of a Thomas splint-cast; however, disadvantages include possible significant injury to the contralateral limb due to increased weight bearing, bandage-associated sores and malunion. ${ }^{11}$ Difficulties in patient activity restriction and bandage monitoring may increase the risk of splint or cast failure. An early return to weight bearing is also more commonly observed in patients with internal plate fixation than those managed conservatively. ${ }^{15}$ 
The authors believe that the slender nature of the ulna in the sheep prohibited the ability to apply plate fixation caudally or laterally from the olecranon to the proximalmid diaphysis. Implant selection that is strong enough to withstand ongoing cyclic fatigue would require greater bone stock distally. Further, orthopaedic wire anchored distally through a transversely drilled hole in the proximal diaphysis with a reduced medullary canal diameter would likely create a stress riser effect and predispose to fracturing through this anchor site. These factors contributed to the selection of a biomechanically robust implant, the String of Pearls locking plate system, with the capability to contour the implant to the sites of greatest bone stock while bridging the fracture gap. In this instance, these sites were the proximal radius and proximal olecranon. The implant was reinforced with cortical screws applied in lag fashion across the fracture line and an intramedullary pin to assist with resist bending forces. These adjuvant implants will likely reduce the cyclic load applied to the plate. Olecranon fracture repair by plate fixation in horses involves the application of a locking compression or dynamic compression plate caudally from the most proximal aspect of the olecranon distally to beyond the transition from ulna to radius, with caudally inserted screws ideal for rigid fixation in adult horses. ${ }^{21}$ Inadvertent penetration of the lateral cortex of the radius with locking head screws is a possible complication following caudal plate application ${ }^{21}$ and may result in devastating radial fracture and subject these patients to euthanasia. In the authors' opinion, the lateral application of the plate improves accuracy of central screw insertion through increased bone stock, thereby reducing the likelihood of implant-associated complications.

In this report, a screw was inadvertently inserted through the proximal ulna epiphyseal plate; however, this is not believed to be of clinical significance given the reduced remaining longitudinal growth expected from this growth plate. A limb length discrepancy was not apparent on followup examination. Plate application to the caudal aspect of the olecranon often avoids soft tissue disruption over the proximal olecranon ${ }^{22}$ but is associated with no biomechanical superiority when compared with lateral plating. The authors elected a lateral approach, sacrificing some soft tissue to improve access to the points of greatest bone stock and apply a more inherently stable implant. The patient demonstrated a satisfactory clinical outcome with no overt pain or lameness apparent at 6 months postoperatively.

\section{Conclusion}

This report illustrates a successful functional outcome of an intra-articular olecranon fracture in a lamb repaired using a String of Pearls locking plate system, intramedullary pin and lag screws. To the authors' knowledge, this is the first report to describe an olecranon fracture and its subsequent repair in the sheep utilizing a novel fixation method. This report further documents the importance of proper case selection and appropriate surgical planning when treating olecranon fractures.

\section{Author Contributions}

All authors contributed to conception of study, study design, acquisition of data and data analysis and interpretation. All authors also drafted, revised and approved the submitted manuscript.

\section{Funding}

None.

\section{Conflict of Interest}

There is no financial or other conflict of interest of any author related to a company or product used in the report.

\section{Acknowledgment}

The authors would like to thank Suzanna M. Abel for providing the figures that demonstrate the anatomy of the antebrachium of sheep (-Fig. 1). These images are Copyright of Suzanne M. Abel.

\section{References}

1 Fox DB. Radius and ulna. In: Tobias KM, Johnston SA, eds. Veterinary Surgery: Small Animals. 1. St. Louis, MO: Elsevier Saunders; 2012:760-784

2 Muir P, Johnson KA. Fractures of the proximal ulna in dogs. Vet Comp Orthop Traumatol 1996;9(02):51-57

3 Fournet A, Boursier JF, Corbeau S, et al. Stabilization of olecranon fractures by tension band wiring or plate osteosynthesis: a retrospective study of 41 cases. Vet Comp Orthop Traumatol 2018;31(01): 53-61

4 Watkins JP. Radius and ulna. In: Auer JA, Stick JA, eds. Equine Surgery. 5th edition. St. Louis, MO: Saunders Elsevier; 2012: 1363-1378

5 Denny HR, Barr AR, Waterman A. Surgical treatment of fractures of the olecranon in the horse: a comparative review of 25 cases. Equine Vet J 1987;19(04):319-325

6 Donecker JM, Bramlage LR, Gabel AA. Retrospective analysis of 29 fractures of the olecranon process of the equine ulna. J Am Vet Med Assoc 1984;185(02):183-189

7 Swor TM, Watkins JP, Bahr A, Honnas CM. Results of plate fixation of type $1 \mathrm{~b}$ olecranon fractures in 24 horses. Equine Vet J 2003;35 (07):670-675

8 Jackson M, Kummer M, Auer J, Hagen R, Fuerst A. Treatment of type 2 and 4 olecranon fractures with locking compression plate osteosynthesis in horses: a prospective study (2002-2008). Vet Comp Orthop Traumatol 2011;24(01):57-61

9 Hague BA, Watkins JP, Hooper RN, Taylor TS, Roussel AJ. Tension band plating of an olecranon fracture in a bull. J Am Vet Med Assoc 1997;211(06):757-758

10 Dyce KM, Sack WO, Wensing CJ, eds. Textbook of Veterinary Anatomy. 4th edition. St. Louis, MO: Saunders Elsevier; 2010

11 Anderson DE, St Jean G. Management of fractures in field settings. Vet Clin North Am Food Anim Pract 2008;24(03):567-582, viii

12 AdamiakZ. Use of semicircular external fixators to treat tibial, radial and ulnar fractures in sheep. Vet Rec 2010;166(11):335-337

13 Anderson DE, St-Jean G. Repair of fractures of the radius and ulna in a ewe using positive profile transfixation pins and casting. Can Vet J 1993;34(11):686-688

14 King GJ, Lammens PN, Milne AD, Roth JH, Johnson JA. Plate fixation of comminuted olecranon fractures: an in vitro biomechanical study. J Shoulder Elbow Surg 1996;5(06):437-441

15 Swor TM, Watkins JR, Bahr A, Epstein KL, Honnas CM. Results of plate fixation of type 5 olecranon fractures in 20 horses. Equine Vet J 2006;38(01):30-34 
16 Newman SD, Mauffrey C, Krikler S. Olecranon fractures. Injury 2009;40(06):575-581

17 Duckworth AD, Clement ND, Aitken SA, Court-Brown CM, McQueen MM. The epidemiology of fractures of the proximal ulna. Injury 2012;43(03):343-346

18 Rommens PM, Küchle R, Schneider RU, Reuter M. Olecranon fractures in adults: factors influencing outcome. Injury 2004;35 (11):1149-1157

19 Baxter G. Surgical Treatment of Equine Ulnar Fractures. Compendium Equine 2008;3(03):147-150
20 Martin F, Richardson DW, Nunamaker DM, Ross MW, Orsini JA. Use of tension band wires in horses with fractures of the ulna: 22 cases (1980-1992). J Am Vet Med Assoc 1995;207(08):1085-1089

21 Kuemmerle JM, Kühn K, Bryner M, Fürst AE. Equine ulnar fracture repair with locking compression plates can be associated with inadvertent penetration of the lateral cortex of the radius. Vet Surg 2013;42(07):790-794

22 Jacobs CC, Levine DG, Richardson DW. Use of locking compression plates in ulnar fractures of 18 horses. Vet Surg 2017;46(02): 242-248 